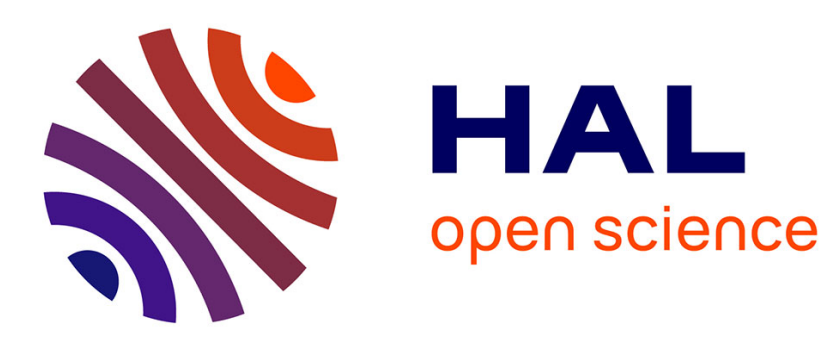

\title{
Cytopathologie ultrastructurale du virus latent de l'artichaut chez Nicotiana benthamiana
}

Auguste Migliori, Jean-Pierre Gourret

\section{To cite this version:}

Auguste Migliori, Jean-Pierre Gourret. Cytopathologie ultrastructurale du virus latent de l'artichaut chez Nicotiana benthamiana. Agronomie, 1987, 7 (2), pp.87-94. hal-00884972

\section{HAL Id: hal-00884972 \\ https://hal.science/hal-00884972}

Submitted on 1 Jan 1987

HAL is a multi-disciplinary open access archive for the deposit and dissemination of scientific research documents, whether they are published or not. The documents may come from teaching and research institutions in France or abroad, or from public or private research centers.
L'archive ouverte pluridisciplinaire HAL, est destinée au dépôt et à la diffusion de documents scientifiques de niveau recherche, publiés ou non, émanant des établissements d'enseignement et de recherche français ou étrangers, des laboratoires publics ou privés. 


\title{
Cytopathologie ultrastructurale du virus latent de l'artichaut chez Nicotiana benthamiana
}

\author{
Auguste MIGLIORI \& Jean-Pierre GOURRET (*) \\ I.N.R.A., Station de Pathologie Végétale, Centre de Recherches de Rennes, F 35650 Le Rheu \\ (*) Laboratoire de Biologie Cellulaire, UA C.N.R.S., $n^{\circ} 256$, Université de Rennes 1, F 35042 Rennes Cedex
}

L'étude au microscope électronique des coupes fines de Nicotiana benthamiana Domin. infecté par le virus latent de l'artichaut (ALV) confirme et augmente les connaissances relatives à la cytopathologie de ce potyvirus. Au stade étudié, après double fixation glutaraldéhyde-acide osmique, le couple $N$. benthamiana ALV se distingue par la présence de 4 constituants absents des cellules saines. (a) Plusieurs vacuoles secondaires sont apparues autour de la vacuole centrale. (b) Des amas paracristallins de virions sont accolés sur toute leur longueur au tonoplaste des vacuoles. Dans les brides cytoplasmiques séparant ces vacuoles les particules virales forment le plus souvent une monocouche ou plus rarement une couche double ou triple. (c) Les " inclusions cylindriques » (sensu Edwardson) se présentent sous la forme d'importants feuillets plurilamellaires (laminated aggregates) et de roues-à-aubes (pinwheels) comprenant le plus souvent 9 aubes faiblement incurvées. Cette structure et l'absence de rouleaux (scrolls) permet d'inclure cet ALV dans la subdivision II de la typologie d'Edwardson. (d) Des tubules de section circulaire de $80 \mathrm{~nm}$ de diamètre existent dans les cellules infectées et sont généralement associés aux groupes de virions. La cytopathologie du couple ALV-N. benthamiana est originale sur plusieurs points (tubules de $80 \mathrm{~nm}$, arrangement paracristallin des virions, importance des feuillets plurilamellaires). Elle devrait s'avérer utile dans le diagnostic viral.

Mots clés additionnels : $A L V$, potyvirus, diagnose, tubules, inclusions.

An electron microscopy study of thin sections from Nicotiana benthamiana Domin. infected with artichoke latent virus (ALV) corroborates and extends known data on the cytopathology of this potyvirus. At the stage studied, after double fixation with glutaraldehyde and osmium, $N$. benthamiana cells infected with ALV showed four features which do not occur in healthy cells : (a) many secondary vacuoles are present around the central vacuole ; (b) paracrystalline arrays of virus particles lie in parallel arrangement against the cytoplasmic face of the tonoplasts (inside the cytoplasmic bridles which separate vacuoles, virus particles generally form a monolayer and more rarely a double or triple layer) ; (c) cylindrical inclusions (sensu Edwardson) appear as thick and large laminated aggregates and as pinwheel arms that often include nine slightly curved blades (this structure and the absence of scrolls sustains assigment of ALV to Edwardson's subdivision II) ; (d) cylindrical tubules, $80 \mathrm{~nm}$ in diameter, occur in infected cells and are most often associated with the paracrystalline arrays of virus particles. The ultrastructural cytopathology of ALV in $N$. benthamiana had several characteristic features $(80 \mathrm{~nm}$ tubules, paracrystalline arrangement of virus particles, importance of laminated aggregates), which may prove useful for diagnosis.

Additional key words : $A L V$, potyvirus, diagnosis, tubules, inclusions.

\section{INTRODUCTION}

Le virus latent de l'artichaut (Artichoke latent virus $=$ ALV) est un virus flexueux appartenant au groupe des potyvirus (RANA et al., 1982 ; MIGLIORI et al., 1984).

Ce virus est fréquent dans les cultures d'artichaut de Bretagne. La détection biologique (réactions des hôtes différentiels) et sérologique (test ELISA =
Enzyme Linked Immunosorbent Assay) a permis de révéler le virus dans 98 à 100 p. 100 des échantillons éprouvés.

La présence quasi générale de l'ALV dans les cultures et l'observation d'inclusions intéressantes dans les cellules infectées nous ont conduit à poursuivre une étude cytopathologique plus approfondie. Les résultats obtenus sont discutés, notamment par rapport aux subdivisions des potyvirus mentionnées par EDWARDSON et al., 1984. 


\section{MATÉRIEL ET MÉTHODES}

L'ALV provenant d'artichaut var " camus de Bretagne » non contaminé par d'autres virus est isolé chez Chenopodium amaranticolor Coste et Reyn, la sélection du virus est effectuée par 5 passages successifs à $C$. amaranticolor (1 passage correspond à un prélèvement d'une lésion locale). Après vérification de la pureté de l'ALV la multiplication est assurée chez Nicotiana benthamiana Domin.

\section{A. Microscope électronique}

Des échantillons de $N$. bentamiana sain ou infecté par l'ALV sont fixés au glutaraldéhyde 2,5 p. 100

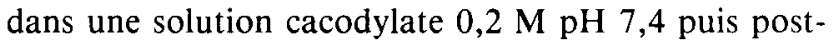
fixés à $4{ }^{\circ} \mathrm{C}$ au tétraoxide d'osmium 1 p. 100 . Après déshydratation à l'éthanol, suivi d'un passage à l'oxyde de propylène, les échantillons sont imprégnés d'un mélange Epon-Araldite (MOLLENHAUER, 1964) qui est soumis à la polymérisation. Les coupes ultrafines obtenues à l'ultramicrotome sont colorées à l'acétate d'uranyle et au citrate de plomb et observées au microscope électronique JEOL $100 \mathrm{CX}$.

La platine goniométrique a été utilisée pour apprécier la structure tridimensionnelle des inclusions et des amas de virions. Des coupes semi-fines de $1 \mu \mathrm{m}$ d'épaisseur ont été effectuées dans tous les échantillons inclus. Ces coupes sont colorées au bleu de toluidine et observées au microscope photonique.

\section{RÉSULTATS}

Nous avons jugé utile de préciser la terminologie établie par EDWARDSON pour décrire les inclusions des potyvirus (fig. 1).

La microscopie électronique confirme le caractère systémique de l'infection. A des degrés différents toutes les cellules contiennent des inclusions et des virions (fig. 2 à 13). Les amas d'inclusions les plus importants se rencontrent dans les cellules du parenchyme chlorophyllien. Les organites cellullaires qui ne montrent pas de différences ultrastructurales par rapport au témoin sain sont les parois, membrane cytoplasmique, noyau, plastes, reticulum, dictyosomes et peroxysomes. Si les mitochondries semblent parfois fusionnées et perturbées (fig. 10) c'est surtout le vacuome qui est modifié par la présence de plusieurs vacuoles secondaires séparées de la vacuole principale par des brides cytoplasmiques (fig. 5, 6, 7, 10,11 et 12). Au contact des tonoplastes, dans le cytoplasme, en particulier au niveau des brides existent des filaments de 12 à $15 \mathrm{~nm}$ de diamètre accolés sur toute leur longueur à la membrane vacuolaire. Ces filaments très denses aux électrons sont entourés d'un halo plus clair d'environ $10 \mathrm{~nm}$ d'épaisseur. En se situant à égale distance les uns des autres (33-36 nm environ de distance centre-àcentre) et en formant plusieurs couches alignées en quinquonce, ces filaments montrent un arrangement très régulier de nature paracristalline. Le nombre de filaments constitutifs des couches décroît au fur et à mesure que l'on s'éloigne du tonoplaste, produisant

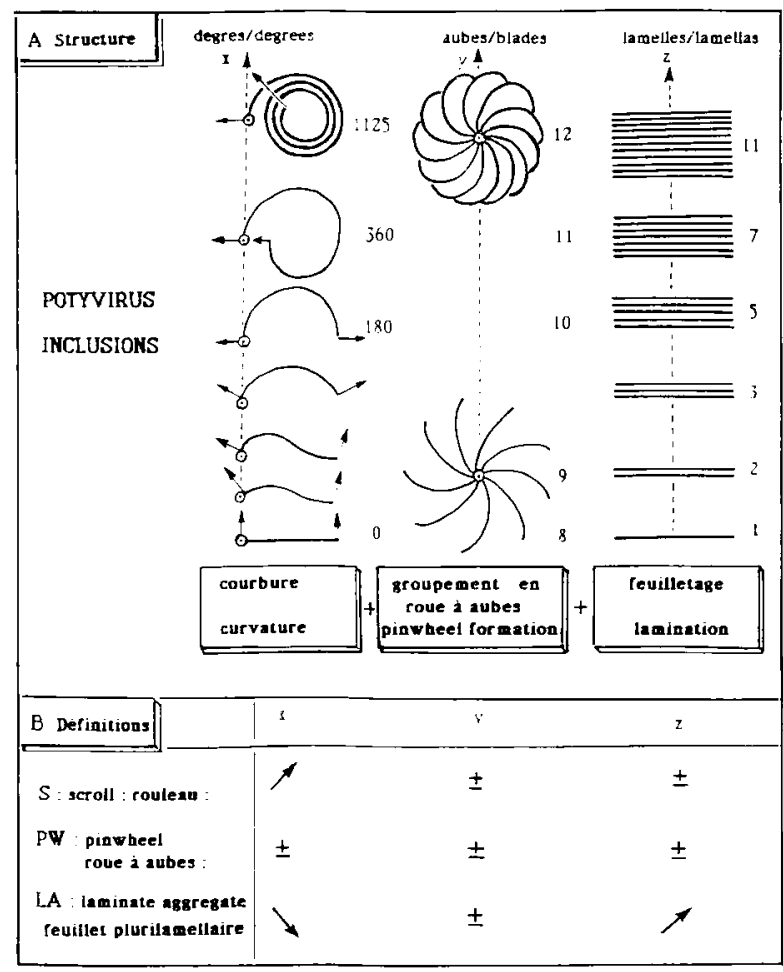

Figure 1

Précisions terminologiques relatives aux inclusions des potyvirus.

Pour faciliter la description de ces assemblages complexes on peut les considérer comme des groupements de lamelles. La lamelle est prise comme unité structurale de base. Outre la forme plane de la lamelle déroulée, il est possible d'utiliser trois paramètres morphologiques: la courbure, le groupement en roue à aubes (nombre d'aubes sur une roue complète et régulière), le feuilletage (nombre de lamelles empilées par feuillet). En A ci-dessus les inclusions sont supposées être coupées perpendiculairement à leur axe $(\odot)$. La courbure peut être définie comme la rotation d'un vecteur orthogonal à la lamelle et se déplaçant du bord axial jusqu'au bout extérieur. Dans un rouleau (S) la courbure est supérieure à $360^{\circ}$ (exemple représenté $:(3 \times 360)+45=1125 \%$. Dans une roue à aubes complète et régulière $(P W)$ la courbure est inférieure à $360^{\circ}$. Elle est nulle ou presque nulle dans un feuillet plurilamellaire $(L A)$. Une courbure moyenne peut être calculée pour une inclusion donnée ou pour les inclusions d'un certain potyvirus. La subdivision I de la typologie d'Edwardson correspond à une courbure très élevée (/) et la subdivision II à une très faible courbure $\backslash$ ).

Terminological details on potyvirus inclusions.

To facilitate the description of these complex structures, let us consider them as arrays of lamella. The lamella is taken as the basic unit. Beyond the planar shape of the unrolled lamella, one may use three morphological parameters: curvature, pinwheel formation (number of blades over a complete and regular wheel), lamination (number of lamella per aggregate). In part $A$ above, inclusions are supposed to be cut perpendicular to their axis $(\odot)$. The curvature is defined through the angular rotation of a vector orthogonal to the lamella and moving over one side from the axial edge of the lamella to the opposite or outer edge. In a scroll (S) the curvature is more than 360 degrees (example in the picture $(3 \times 360)+45=$ 1125 degrees). In pinwheels $(P W)$ the curvature is less than 360 degrees. It is almost equal to zero in laminated aggregates (LA). A mean curvature can be defined for a particular inclusion or for the overall inclusions of a specific potyvirus. Subdivision I in Edwardson's typology of the inclusions corresponds to the highest curvature ( $/$ ) while subdivision II corresponds to the lowest $(\backslash$ ).

ainsi une sorte d'arrangement en gradins (fig. 9). Dans ces agrégats les extrémités des filaments coïncident (fig. 3 et 4). La rareté des coupes parfaitement longitudinales passant par ces agrégats et l'incertitude que l'on a sur l'orientation de coupes approchant 


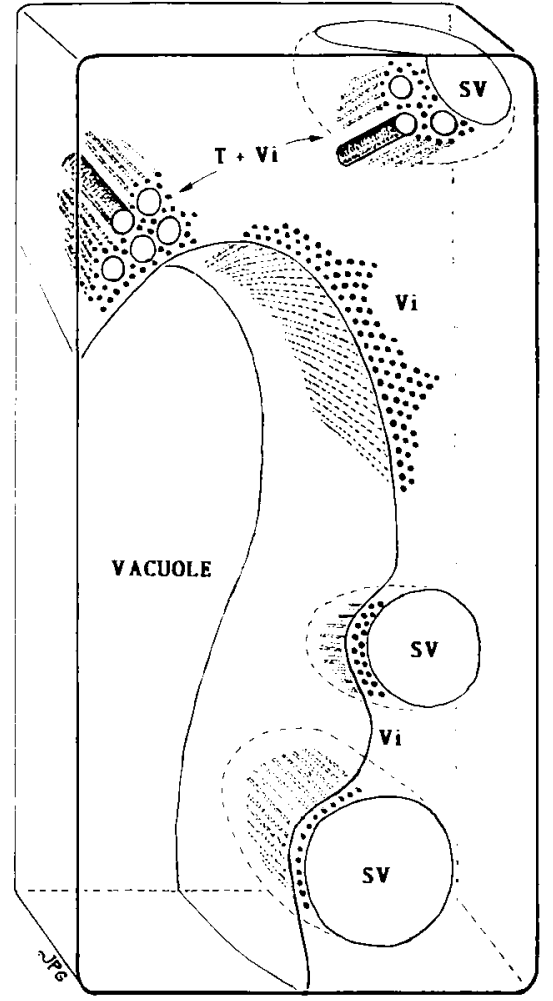

Figure 2

Schéma des tubules et des arrangements paracristallins des virions de l'ALV dans les cellules infectées de Nicotiana benthamiana.

Schematic view of tubules and paracrystalline arrays of ALV particles in infected cells of Nicotiana benthamiana. tubules rectilignes existent aussi parfois isolément et sans orientation précise dans le hyaloplasme (fig. 10) ou bien ils forment des faisceaux. Leur longueur n'a pu être déterminée sur les coupes.

Le hyaloplasme des cellules de $N$. benthamiana infecté par l'ALV renferme d'abondantes inclusions caractéristiques des potyvirus, très nettement visibles en microscopie photonique. Elles apparaissent dans les coupes-fines sous forme d'étoiles ou de faisceaux fortement colorés au bleu de toluidine. Au microscope électronique à transmission, ces inclusions se révèlent toutes constituées de lamelles d'épaisseur constante, empilées et associées par leur bord en assemblages caractéristiques (fig. 13). Ces inclusions sont libres dans le hyaloplasme ; elles ne sont pas liées à la membrane cytoplasmique. La platine goniométrique a permis de les photographier sous un angle qui facilite leur compréhension et leur description. Plusieurs aspects de leur morphologie mérite d'être soulignés. En adoptant la terminologie d'EDWARDSON, on note l'importance en surface et en épaisseur des feuillets plurilamellaires (laminated aggregates notés LA dans les clichés et schémas), la présence de roues-à-aubes (pinwheels sensu stricto notés $\mathrm{PW}$ ) et l'absence de rouleaux (scrolls). Les feuillets plurilamellaires LA regroupent souvent près de 10 lamelles et parfois davantage. Ces lamelles de 5 à $6 \mathrm{~nm}$ d'épaisseur sont l'unité structurale de base du système. Leur courbure moyenne, définie dans la figure 1, est faible. Il a été possible de déterminer, sur une trentaine de cas, le nombre de feuillets soudés par leur bord à l'axe de la roue à aubes. Le plus fréquemment, le nombre de ces « aubes » est égal à neuf.

\section{DISCUSSION}

La formation de vacuoles secondaires a déjà été observée et analysée dans le cas de potyvirus (MARTELLI et al., 1969). Cependant que d'autres auteurs notent le fait en mentionnant la présence de brides cytoplasmiques où s'accumulent les particules virales (RUSSO \& MARTELLI, 1969 ; KIM \& FULTON, 1969 ; HOEFERT, 1969 ; WEINTRAUB \& RAGETLI, 1970 ; BuVAT, 1974 ; GoURret \& Triharso, 1977). Cette observation est donc fréquente chez les potyvirus.

Les filaments de $12-15 \mathrm{~nm}$ de diamètre correspondent très probablement aux virions bien que leur longueur, leur composition, leur antigénicité n'aient pu être déterminées.

Si la proximité des particules virales par rapport aux tonoplastes a été relatée dans d'autres études cytopathologiques consacrées à des potyvirus (ZETTLER et al., 1968 ; KIM \& FULTON, 1969 ; RUSSO \& MARTELli, 1969 ; MARTELli et al., 1969 ; WEINTRAUB \& RAGETLI, 1970 ; BUVAT, 1974 ; VERHOYEN et al., 1976 ; TEAKLE \& PARES, 1977) c'est la première fois à notre connaissance qu'un arrangement paracristallin de potyvirus est observé. Il est à noter qu'une légère torsion affecte les virions les uns par rapport aux autres. Ce type de torsion se rencontre dans les cristaux liquides ou mésophases cholestériques (BOULIGAND, 1972 et 1984).

Quelle est la composition et la signification des tubules cylindriques de $80 \mathrm{~nm}$ ? BEGTRUP (1976) a cette orientation rend difficile la détermination de la longueur des filaments. Celle-ci serait de l'ordre de $700 \mathrm{~nm}$. Il n'a pas été observé de filaments libres dans le hyaloplasme.

Des tubes cylindriques de 75 à $85 \mathrm{~nm}$ de diamètre sont fréquemment associés aux agrégats de filaments et alignés parallèlement à ceux-ci (fig. 11 et 12). Ces 

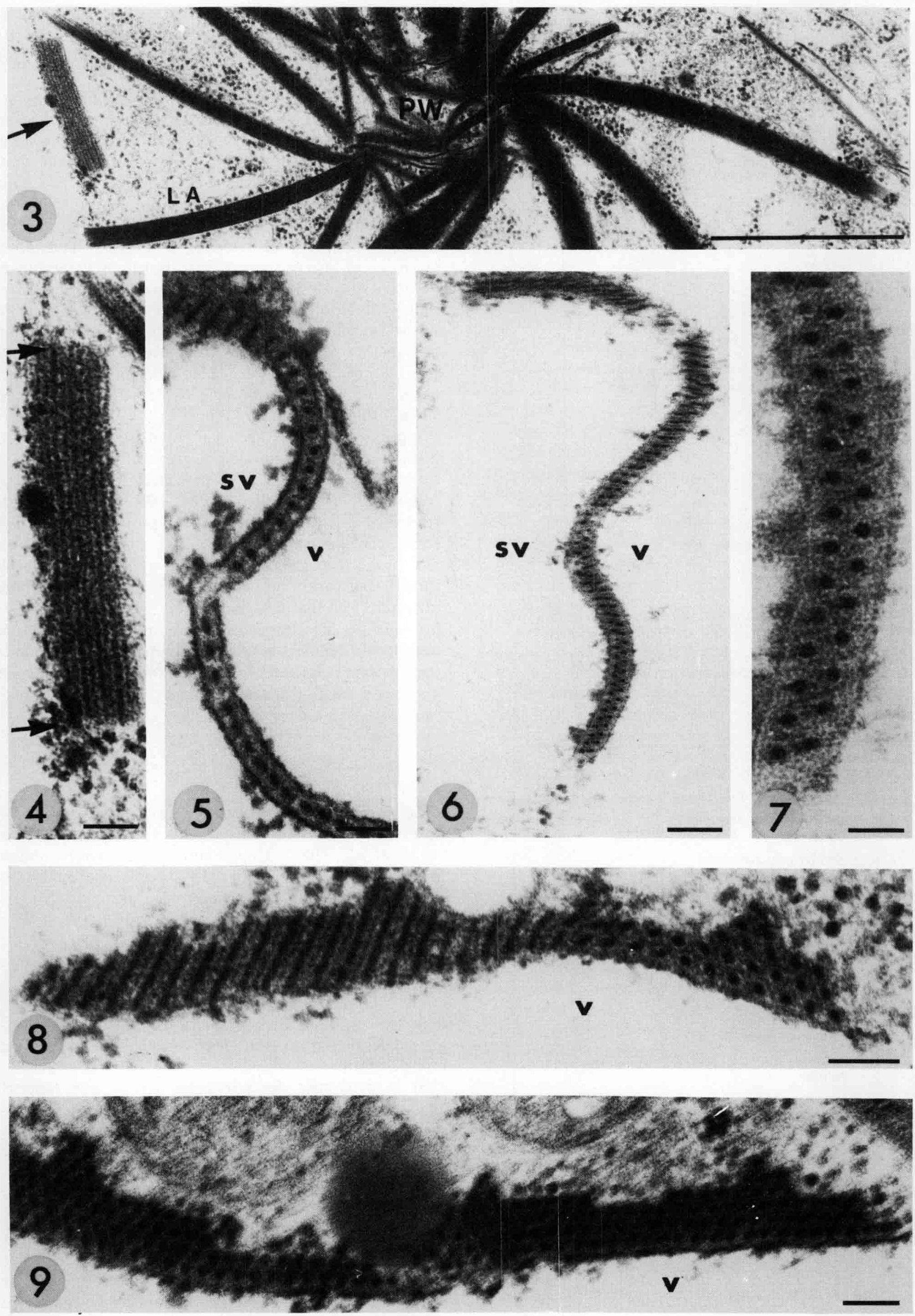

Figure 3 

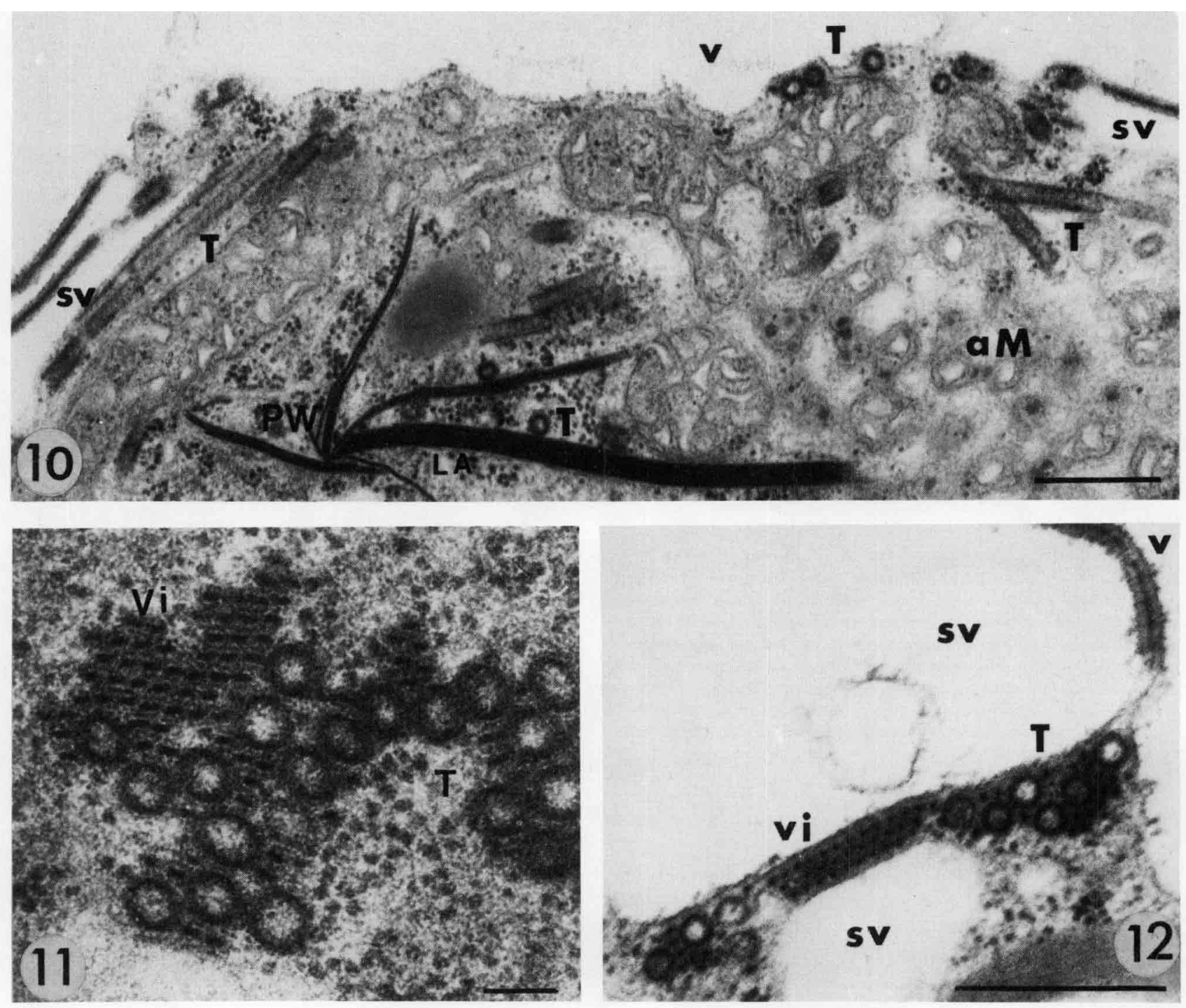

Figure 4

Un agrandissement de la figure 3. Barre: $100 \mathrm{~mm}$.

$A$ higher magnification of figure 3. Bar: $100 \mathrm{~nm}$.

Figure 5

Bride cytoplasmique renfermant une monocouche de virions. Barre : $100 \mathrm{~nm}$. Vacuole (V), vacuole secondaire (SV).

Cytoplasmic bridle containing a monolayer of virus particles. Bar : $100 \mathrm{~nm}$. Vacuoles $(V)$, secondary vacuoles (SV).

Figure 6

Bride cytoplasmique renfermant une double couche de virions. Barre : $200 \mathrm{~nm}$.

Cytoplasmic bridle containing a double layer of virus particles. Bar : $200 \mathrm{~nm}$.

Figure 7

La figure 7 est un agrandissement de la figure 6. Barre : $50 \mathrm{~nm}$.

Higher magnification of figure 6. Bar: $50 \mathrm{~nm}$.

Figure 8

Amas paracristallin de virions montrant la torsion progressive de leur direction. Sur la partie en coupe oblique à gauche, la bande claire de part et d'autre de chaque virion correspond au halo circulaire qui entoure chaque particule vue en coupe transversale. Audelà du halo se situe le hyaloplasme moyennement dense aux électrons. Barre : $10 \mathrm{~nm}$.

Paracrystalline array of virus particles showing a progressive twist of their axis. On the left of the figure, the light bands which lie on both sides of the particles correspond to the circular halo which surrounds each particle in transverse section. Beyond the halo is the moderately electron-dense hyaloplasm. Bar : $100 \mathrm{~nm}$.

Figure 9

Amas paracristallin de virions montrant la disposition « en gradins" des couches successives et l'arrangement en quinquonce. Barre : $100 \mathrm{~nm}$.

Paracrystalline array of virus particles showing a triangular lattice (quincunx). On the cytoplasmic side, the number of particles gradually decreases in tiers. Bar : $100 \mathrm{~nm}$.

Tubules et virions (ALV-N. benthamiana).

Tubules and virus particles (Al.V-N. benthamiana).

Figure 10

Les tubules cylindriques de $80 \mathrm{~nm}$ de diamètre sont vus en coupes transversales, obliques ou longitudinales $(T)$. Noter qu'il n'y a pas de virions à leur contact. aM: Mitochondries anormales, alvéolisées. Barre : $500 \mathrm{~nm}$.

Cylindrical tubules $80 \mathrm{~nm}$ in diameter are seen in transverse. oblique or longitudinal section. No virions appear to be in contact with them. aM : abnormal, alveolar mitochondria. Bar : $500 \mathrm{~nm}$.

Figure 11

Assemblage mixte de tubules (T) et de virions (Vi). Barre: $100 \mathrm{~nm}$. Composite array of tubules (T) and virus particles (Vi). Bar: $100 \mathrm{~nm}$.

Figure 12

Assemblage mixte de tubules (T) et virions (Vi) au contact du vacuome de la cellule infectée. Une partie de la vacuole principale est visible à droite (V). Barre : $500 \mathrm{~nm}$.

Composite array of tubules (T) and virus particles (Vi) associated with secondary vacuoles $(S V)$. Part of the central vacuole appears on the right (V). Bar : $500 \mathrm{~nm}$. 


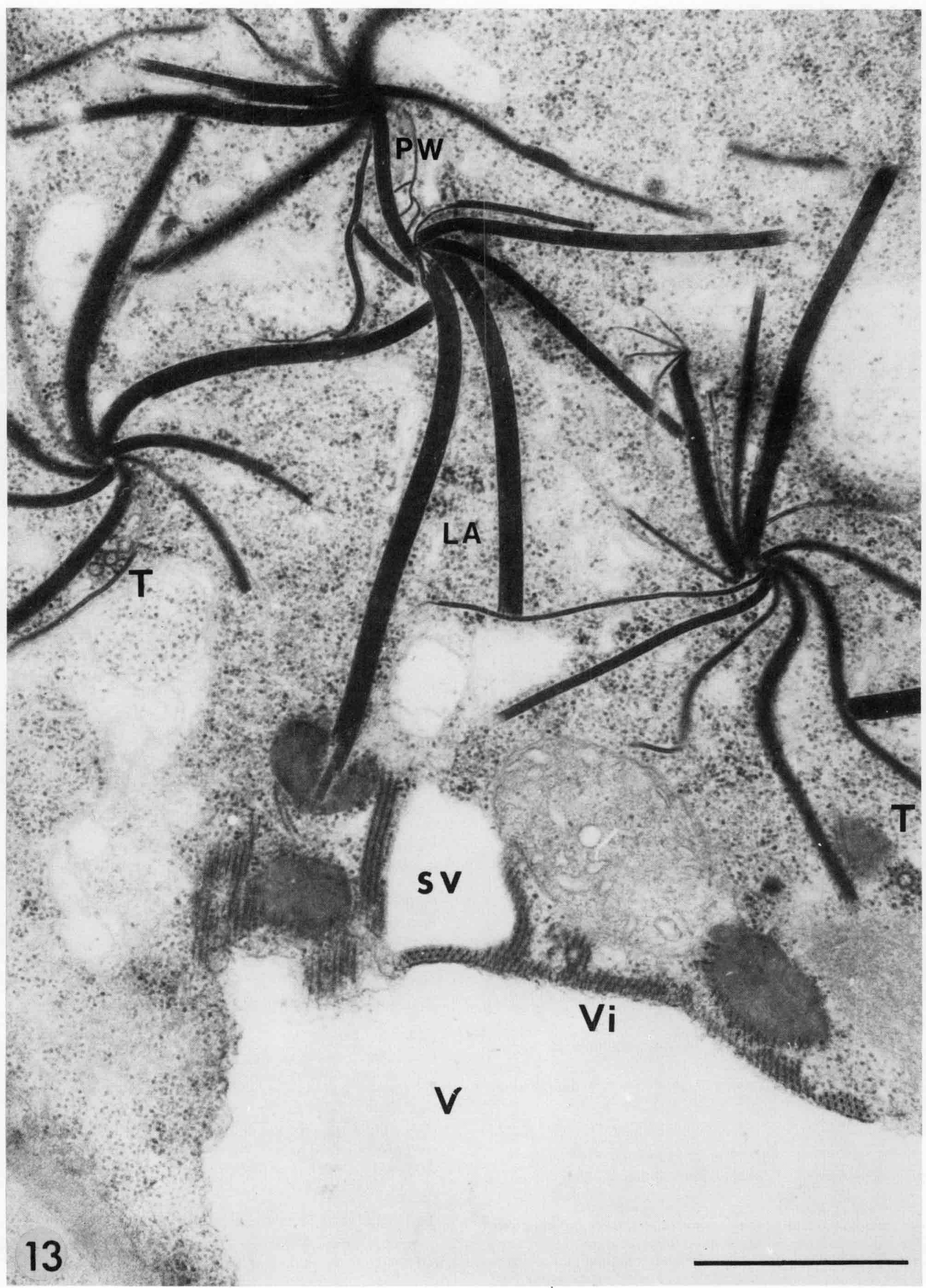

Figure 13

Vue générale des inclusions et des virions ( $A L V$-N. benthamiana). Noter la morphologie des inclusions et la position juxtavacuolaire des groupes de virions, (Vi). T: tubules en section transversale. Barre : $1000 \mathrm{~nm}$.
General view of inclusions and virus particles $(A L V-\mathrm{N}$. benthamiana). Note the morphology of the inclusions and the juxtavacuolar location of virus particle arrays, (Vi). T: transverse section of tubules. Bar : $1000 \mathrm{~nm}$. 
noté une association de microtubules de $20 \mathrm{~nm}$ avec les feuillets plurilamellaires (LA) d'un potyvirus. De même une association paracristalline régulière de cylindres creux d'environ $60 \mathrm{~nm}$ et de virions appartenant à un potyvirus, a été observée (GOURRET, 1975). Dans ce cas les cylindres étaient de nature membranaire et dérivaient du reticulum endoplasmique. Dans le cas présent, les tubules de $80 \mathrm{~nm}$ sont ouverts aux extrémités et ne semblent pas être formés par une membrane. Ces tubules qui pourraient résulter d'une réorganisation de protéines cellulaires préexistant à l'infection virale ou de matériaux néosynthétisés sont un élément nouveau et intéressant de la cytopathologie du couple ALV-N benthamiana. En raison de leur diamètre et de leur structure ces tubules ne peuvent être confondus avec les rouleaux ou scrolls que forment de nombreux potyvirus (EDWARDSON, 1966). Ils diffèrent aussi des tubules de 20-22 nm de diamètre signalés dans le cas de la mosaïque de la Betterave (BMV), (RUSSO \& MARTELli, 1969 ; MARTElli et al., 1969).

Le couple ALV-N. benthamiana semble être un bon modèle pour étudier la géométrie et la dynamique de formation des inclusions dites « cylindriques ». Les aubes en feuillets plurilamellaires ont une épaisseur suffisante pour être vues en microscopie photonique, facilitant ainsi une reconstitution tridimensionnelle complète de plusieurs inclusions au moyen de coupes sériées et de la platine goniométrique. Le nombre d'aubes égal à 9 se retrouve fréquemment dans les études de cytopathologie des potyvirus. On peut se demander si ce nombre dépend de la plante-hôte ou du génome viral. En ce qui concerne la courbure des lamelles, ce type de question a été tranché en faveur des gènes viraux (EDWARSON, 1974 ; EDWARDSON et al., 1984).

EDWARDSON \& CHRISTIE (1978) ont montré l'intérêt des inclusions induites par les virus dans la classification et diagnostic viral. D'autres travaux confortent ce raisonnement dans le cas de potyvirus (Mc DONALD \& Hiebert, 1974 ; Chamberlain \& Cathrall, 1977 ; Mernaugh et al., 1980). Les inclusions "cylindriques" par EDWARDSON (1968) sont ici représentées par des roues à aubes (pinwheels $\mathrm{PW}$ ) et par d'abondants feuillets plurilamellaires. Cet assortiment d'inclusions permet de rattacher l'ALV étudié à la subdivision II de la typologie établie par EDWARDSON (1974 et complétée en 1984). RANA et al., 1982, aboutissaient à la même conclusion. Les autres aspects des inclusions observées dans le couple ALV$N$. benthamiana permettent-ils de préciser la diagnose ? Il est raisonnable de penser que les tubules de $80 \mathrm{~nm}$ et la structure paracristalline des groupements de virions puissent permettre d'affiner la diagnose si l'on associe des critères cytologiques à l'étude sérologique des inclusions. Les propriétés antigéniques des inclusions ont en effet été utilisées avec succès par YeH \& GONSAlves (1984); PURCIFUll et al. (1984a et $b$ ) pour la différenciation des potyvirus.

Reçu le 29 avril 1986. Accepté le 8 octobre 1986.

\section{RÉFÉRENCES BIBLIOGRAPHIQUES}

Begtrup J., 1976. Tubular structures in the cytoplasm of mesophyll cells in plants infected with carnation vein mottle virus. Phytopathol. Z., 86, 127-135.

Bouligand Y., 1972. Twisted fibrous arrangements in biological materials and cholesteric mesophases. Tissue \& cell, 4, 189-217.

Bouligand Y., 1984. Cristaux Liquides. In Encyclopoedia universalis, 754-759.

Buvat R., 1974. Structures cytoplasmiques apparemment induites par un virus dans les faisceaux conducteurs d'entrenœuds de Cucurbita pepo L. (Cucurbitaceae). C.R. Acad. Sci., Paris, 278, 31953198.

Chamberlain J. A., Cathrall P. L., Jellings A. J., 1977. Symptoms and electron microscopy of ryegrass mosaic virus in different grass species. J. gen. Virol., 36, 297-306.

Edwardson J. R., 1968. Electron microscopy of cytoplasmic inclusions in cells infected with rod-shaped viruses. Am. J. Bot., 53, 359364.

Edwardson J. R., 1966. Cylindrical inclusions in the cytoplasm of leaf cells with tobacco etch virus. Science, 153, 883-884.

Edwardson J. R., 1974. Some properties of the potato virus $Y$ group. Fla. Agric. Exp. Stn. Monogr., 4, 398.

Edwardson J. R., Christie R. G., 1978. Use of virus-induced inclusions in classification and diagnosis. Ann. Rev. Phytopathol., 16, 31-35.

Edwardson J. R., Christie R. G., Ko N. J., 1984. Potyvirus cylindrical inclusions. Subdivision IV. Phytopathology, 74, 1111-1114.

Gourret J. P., 1975. Une étude ultrastructurale comparative des Mycoplasmes phytopathogènes, des bactéries des nodules racinaires et des plastes. Thèse d'Etat C 207-59, Rennes 1975. AO CNRS 13196.
Gourret J. P., Triharso, 1977. Etude ultrastructurale d'une virose et d'une mycoplasmose d'Arachis hypogaea L. C.R. Acad. Sci., Paris, sér. D, 284, 179-182.

Hoefert L. L., 1969. Proteinaceous and virus-like inclusions in cells infected with beet mosaic virus. Virology, 37, 498-501.

Kim K. S., Fulton J. P., 1969. Electron microscopy of pokeweed leaf cells infected with pokeweed mosaic virus. Virology, 37, 297308.

Martelli G. P., Russo M., Castellano M. A., 1969. Ultrastructural features of Malva parviflora L. with vein clearing and of plants infected with beet mosaic virus. Phytopathol. mediterr, 8, 175-186.

Mc Donald J. G., Hiebert E., 1974. Ultrastructure of cylindrical inclusions induced by viruses of the potato $Y$ group as visualized by freeze-etching. Virology, 58, 200-208.

Mernaugh R. L., Gardner W. S., Yocom K. L., 1980. Three dimensional structure of pinwheel inclusions as determined by analytical geometry. Virology, 106, 273-281.

Migliori A., Lot H., Pecaut P., Duteil, Rouze-Jouan J., 1984. Les virus de l'artichaut. I. Mise en évidence de trois virus dans les cultures françaises d'artichaut. Agronomie, 4, 257-268.

Mollenhauer H. H., 1964. Plastic embedding mixtures for use in electron microscopy. Stain Technology, 39, 111-114.

Purcifull D., Edwardson J., Hiebert E., Gonsalves D., 1984a Papaya ringspot virus C.M.I./A.A.B. Description of Plant Viruses. $\mathrm{N}^{\circ} 292$.

Purcifull D., Hiebert E., Edwardson J., 1984b. Watermelon mosaic virus 2 C.M.1./A.A.B. Description of Plant Viruses. $N^{\circ} 293$.

Rana G. L., Russo M., Gallitelli D., Martelli G. P., 1982. Artichoke latent virus : characterization, ultrastructure and geographical distribution. Ann. Appl. Biol., 101, 279-289. 
Russo M., Martelli G. P., 1969. Cytopathology of Gomphrena globosa L. plants infected by beet mosaic virus. Phytopathol. mediterr, 8, 65-82.

Teakle D. S., Pares R. D., 1977. Potyvirus (Potato virus Y) group In Atlas of Insect and Plant Viruses. Ed. K. Maramorosch, 8, 311-321. Academic Press.

Verhoyen M., Esparza-Duque J., Matthieu J. L., Horvat F., 1976 Identification du virus latent du céleri en Belgique. Parasitica, 32, 158-166.

Weintraub M., Ragetli H. W. J., 1970. Distribution of virus like particles in leaf cells of Dianthus barbatus infected with carnation vein mottle virus. Virology, 40, 868-881.
Yeh S. D., Gonsalves D., 1984. Purification and Immunologica Analyses of Cylindrical. Inclusion Protein Induced by Papaya Ringspot Virus and Watermelon Mosaic Virus 1, Phytopathology, 74, $1273-1278$.

Zettler F. M., Edwardson S. R., Purcifull D. E., 1968. Ultramicroscopic differences in inclusions of papaya mosaic virus and papaya ringspot virus correlated with differential aphid transmission. Phytopathology, 58, 332-335. 